\title{
A!
}

This is an electronic reprint of the original article.

This reprint may differ from the original in pagination and typographic detail.

Makkonen, Ilja; Salo, Petri; Alatalo, Matti; Rahman, Talat S.

\section{Ab initio studies of stepped Pd surfaces with and without S}

Published in:

Physical Review B

DOI:

10.1103/PhysRevB.67.165415

Published: 01/01/2003

Document Version

Publisher's PDF, also known as Version of record

Please cite the original version:

Makkonen, I., Salo, P., Alatalo, M., \& Rahman, T. S. (2003). Ab initio studies of stepped Pd surfaces with and without S. Physical Review B, 67, 165415. https://doi.org/10.1103/PhysRevB.67.165415

This material is protected by copyright and other intellectual property rights, and duplication or sale of all or part of any of the repository collections is not permitted, except that material may be duplicated by you for your research use or educational purposes in electronic or print form. You must obtain permission for any other use. Electronic or print copies may not be offered, whether for sale or otherwise to anyone who is not an authorised user. 


\title{
$A b$ initio studies of stepped Pd surfaces with and without $\mathrm{S}$
}

\author{
Ilja Makkonen, Petri Salo, and Matti Alatalo \\ Laboratory of Physics, Helsinki University of Technology, P.O. Box 1100, FIN-02015 HUT, Finland
}

Talat S. Rahman

Department of Physics, Cardwell Hall, Kansas State University, Manhattan, Kansas 66506

(Received 10 July 2002; revised manuscript received 18 December 2002; published 28 April 2003)

\begin{abstract}
We have performed a comprehensive first-principles study of the electronic and geometric structure of a set of clean and S covered vicinal Pd surfaces of (111) and (110), namely $\operatorname{Pd}(211), \operatorname{Pd}(331), \operatorname{Pd}(320)$, and $\operatorname{Pd}(551)$, each having three-atom wide terraces. The trends in the multilayer relaxation patterns can generally be explained on the basis of charge smoothening which makes the vicinals of $\mathrm{Pd}(110)$ prone to larger and deeper relaxations in comparison to the $\mathrm{Pd}(111)$ counterparts. A range of adsorption energies for preferred adsorption sites for $\mathrm{S}$ is found on all four surfaces, with $\mathrm{Pd}(320)$ being the most receptive. We show that the adsorbate can have considerable effect on multilayer relaxations and registry of the substrate atoms. We discuss the nature of the S-Pd bonding and how this bonding is affected by the surface geometry and coordination. We relate the poisoning effect of $S$ to the changes in the electronic structure of the substrate atoms.
\end{abstract}

DOI: 10.1103/PhysRevB.67.165415

PACS number(s): 68.43.-h, 68.47.De, 73.20.Hb

\section{INTRODUCTION}

Advances in experimental and theoretical techniques, supplemented by enhanced computational power, have made it possible for surface scientists to carry the knowledge already gained from studies of homogeneous, flat, low Miller index surfaces to their heterogeneous, stepped, high Miller index counterparts. This venture is not just academically challenging, it also opens many new vistas, in particular, more contact with the real world in which, at the atomic scale, surfaces have defects such as kinks, steps, and vacancies. By examining processes such as adsorption, dissociation, and eventually chemical reactions at steps and kink sites of transition metals, for example, information and insights relevant to industrial catalysts may be obtained. The main challenge, however, is to identify and understand the role played by the local environment in determining the characteristics of the system at the atomic scale. It is thus prudent to carry out systematic and accurate studies of vicinal surfaces that are the high Miller index surfaces, containing regular arrays of monoatomic steps of specific orientation, separated by terraces of specific width and orientation.

Several experimental and theoretical studies ${ }^{1-11}$ have already pointed to the important role played by the step atoms. In short, experimental studies of dissociation of gases such as $\mathrm{O}_{2}, \mathrm{H}_{2}, \mathrm{~N}_{2}$, and $\mathrm{NO}$ on stepped $\mathrm{Pt}, \mathrm{Ag}, \mathrm{Ru}$, and $\mathrm{Pd}$ surfaces, as the case may be, show the process to be significantly advanced in the presence of steps. For example, Dahl et al. claim that $\mathrm{N}_{2}$ dissociation rate on stepped $\mathrm{Ru}(0001)$ is about nine orders of magnitude higher than on the terraces at $500 \mathrm{~K} .{ }^{11}$ Experiments have also pointed to the relative sensitivities of the step and terrace sites for adsorption: Irokawa et al. interpret their ultraviolet photoelectron spectroscopy data $^{8}$ to imply preferential adsorption of NO on terrace sites of $\operatorname{Pd}(211)$ at low coverages and at step sites at higher coverages, in agreement with previous experimental observations. ${ }^{10}$ Theoretical studies based on densityfunctional theory have supported the general conclusion about the importance of steps arising from these and related experimental studies. For instance, the strong sensitivity of the step atoms to $\mathrm{NO}$ bond activation found on $\mathrm{Ru}(0001)$ (Ref. 1), was shown by Hammer, ${ }^{2}$ to be related to the large reduction in the energy barrier for dissociation near step edges as a consequence of the enhanced ability of the reaction products to rebind with the step atoms. Earlier Hammer and Nørskov ${ }^{9}$ had used density-functional theory (DFT) calculations to relate the site preference for adsorption, and subsequent rearrangement at higher coverages for $\mathrm{NO}$ on $\operatorname{Pd}(211)$, to the location of the $d$-band center: The higher the $d$-band center, the stronger they found the chemisorption bond.

It is natural to link the enhanced chemical reactivity of the step atoms to their reduced coordination. The lack of neighbors at the step sites, of course, leads to a redistribution of electron-charge density even in the absence of adsorbates, as was suggested long back by Smoluchowski. ${ }^{12}$ In fact, several experimental and theoretical studies of stepped surfaces of transition metals have already revealed striking trends in their multilayer relaxations. A recent theoretical study ${ }^{13}$ has pointed to the important role played by the atoms just beneath steps in surface relaxations of vicinals of $\mathrm{Cu}(111)$ and $\mathrm{Cu}(100)$. Our interest in this paper is in stepped Pd surfaces that have already been shown to have important site sensitive characteristics for chemisorption of NO (Refs. 8-10) and $\mathrm{H}_{2} \cdot 7,14,15$

Palladium is an important catalyst in different industrial and technological processes. We are thus interested in a systematic study of the surface electronic and geometric structure of a set of vicinal surfaces of Pd. To extract the role of local coordination and environment, we have chosen to examine the characteristics of two vicinals each of $\operatorname{Pd}(100)$ and $\operatorname{Pd}(111)$, each with approximately three-atom wide terraces, and a specific step orientation. Of these, $\operatorname{Pd}(320), \operatorname{Pd}(211)$, and $\operatorname{Pd}(551)$ have already been the subject of several types of experimental observations. ${ }^{16-18}$ While the stability of $\operatorname{Pd}(331)$ has been questioned, ${ }^{19}$ it provides a useful point of 
TABLE I. Surface structures in microfacet notation: ${ }^{33}$ Miller indices $h, k$, and $l ; n$ is the number of terrace atoms in a single step and perpendicular to the step edges; $t$ means terrace and $s$ step facet.

\begin{tabular}{lccc}
\hline $\begin{array}{l}\text { Surface } \\
h k l\end{array}$ & $\begin{array}{c}\text { Microfacet notation } \\
n\left(h_{t} k_{t} l_{t}\right)\left(h_{s} k_{s} l_{s}\right)\end{array}$ & $\begin{array}{c}\text { Miscut angle } \\
\left({ }^{\circ}\right)\end{array}$ & $\begin{array}{c}\text { Step-step distance } \\
(\AA)\end{array}$ \\
\hline 211 & $3(111)(100)$ & 19.5 & 6.86 \\
331 & $3(111)(111)$ & 22.0 & 6.11 \\
320 & $3(110)(100)$ & 11.3 & 7.14 \\
551 & $3(110)(111)$ & 8.0 & 10.00 \\
\hline \hline
\end{tabular}

comparison in order to understand the electronic structures and geometries of the different stepped surfaces. Additionally, since poisoning is one of the negative attributes in catalysis, and $\mathrm{S}$ is well known to act as a poison on, e.g., $\mathrm{Pd}$, $\mathrm{Pt}$, and Ni surfaces, ${ }^{20-25}$ we have undertaken a comprehensive study of $\mathrm{S}$ on $\mathrm{Pd}$ surfaces. The understanding of the poisoning processes may help in the design of better catalysts, where one can possibly avoid the poisoning by tuning the surface either by doping or introducing different steps, kinks, etc., than originally present. To address the poisoning mechanisms for any particular reaction, it is useful to know in detail how the poisoning atoms themselves stick to the surface and whether they alter the surface structure. These studies of the electronic and geometric structure of clean and $\mathrm{S}$ covered, stepped Pd surfaces, are performed through selfconsistent solutions of Kohn-Sham equations ${ }^{26}$ using the DFT with the gradient correction to the local electron densities.

The rest of the paper is organized as follows. In Sec. II, we describe the method used, in Sec. III, we discuss our results for the relaxations of different stepped Pd surfaces and compare them with those of other, related studies. In Sec. IV, the results for the $\mathrm{S}$ adsorption on these surfaces is discussed. Finally, in Sec. V, we provide conclusions.

\section{DETAILS OF THE CALCULATIONS}

All of our calculations are based on $a b$ initio DFT. Here the Kohn-Sham equations were solved in a plane-wave basis using the Vienna $a b$ initio simulation package. ${ }^{27}$ Ultrasoft pseudopotentials of the Vanderbilt type ${ }^{28}$ as implemented by Kresse and Hafner ${ }^{29}$ were used, for which a cutoff energy for the plane-wave expansion of $199 \mathrm{eV}$ was found to give converged total energies for the system. In the Brillouin zone, sampling an $8 \times 8 \times 1$ Monkhorst-Pack mesh ${ }^{30}$ was used, together with a Methfessel-Paxton smearing ${ }^{31}$ of the energy levels $\sigma=0.1 \mathrm{eV}$. Tests using larger $k$-point sets yielded essentially the same results for, e.g., the interlayer relaxations that are known to be rather sensitive to the $k$-point sampling. ${ }^{13}$ The Perdew-Wang functional ${ }^{32}$ was used for the generalized gradient approximation description of the exchange and correlation.

The relaxations of stepped $\mathrm{Pd}$ surfaces and the adsorption of $\mathrm{S}$ on them, were studied using four different high-index surfaces: fcc(211), fcc(331), fcc(320), and fcc(551); see Table I and Fig. 1 for elaboration. The fcc(211) and fcc(331) surfaces are vicinals of fcc(111). If only monoatomic steps and unkinked step edges are considered, these two surfaces are representatives of the two possible classes of the vicinals of fcc(111). The difference between these classes is the structure of the step face. The fcc(211) surface is composed of (111) terraces separated by single-atom-height steps of (100) orientation (the so-called $A$-type step). The structure of the single-atom-height step faces of fcc(331) is fcc(111) (the socalled $B$-type step). The other two surfaces fcc(320) and fcc(551) are two different kinds of vicinals of fcc(110). On fcc(320) the monoatomic steps are along (001), while on fcc(551) they are along (1 10$)$. Thus, the step edge of the latter is close-packed, while that of the former is more open and may be considered as kinked. Together, these four surfaces provide four different local environments, while maintaining only three atoms on each terrace, albeit with different terrace widths.

In the slabs used in the calculations, from 8 to 30 atomic layers are repeated periodically in a supercell geometry with $10 \AA$ vacuum between the slabs. The supercells are only two atoms wide in the direction parallel to the step edges. Except for $\operatorname{Pd}(211)$, for which only one terrace was used, the supercells consist of two terraces. Atoms were placed into their ideal fcc lattice sites and the structure was relaxed until the forces acting on each atom converged better than $0.01 \mathrm{eV} / \AA$. Atoms in the 3-5 bottom layers were kept fixed during relaxation in order to preserve the lattice geometry bulklike several layers away from the surface. The layer thickness was varied to test the convergence of the results for multilayer relaxation. As expected, the vicinals of fcc(110) required larger number of layers to get good convergence. When the adsorption sites of $\mathrm{S}$ were tested, the $\mathrm{S}$ atom was placed into the presumed adsorption site on a relaxed palladium surface, and the whole system was relaxed until the $S$ atom moved away from the site or the forces acting on atoms were smaller than $0.02 \mathrm{eV} / \AA$. In all the calculations, the lattice constant $3.96 \AA$, obtained from bulk calculations, was used.

\section{ENERGETICS AND RELAXATIONS OF STEPPED Pd SURFACES}

The observed enhanced catalytic activity at the steps is presumably due to the rearrangement of the electroniccharge density in the vicinity of the steps which naturally affects the atomic geometries at the step. Therefore, the understanding of atomic relaxations at the steps serves as a useful starting point for the interpretation of reactions and poisoning occuring on stepped surfaces. Towards this end, we have calculated the relaxations of the above-mentioned stepped surfaces, first without adsorbates. The percentage changes in the interlayer spacings are shown in Table II. As a prototype, the definitions of the different layers, together with the corresponding relaxation, are shown for $\mathrm{Pd}(211)$ in Fig. 2. It can be seen in the figure that the layers labeled 1, 2, and 3 are exposed to the vacuum, while the others are not. These are the three undercoordinated sites on $\operatorname{Pd}(211)$ with coordination 7, 9, and 10, respectively. In the case of $\mathrm{Pd}(331)$, there are also three undercoordinated sites on the terrace with coordinations 7,9 , and 11 . On the other two 
(a)

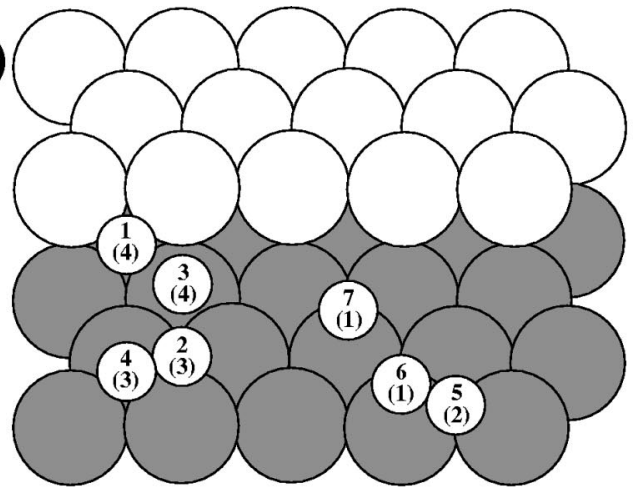

(c)

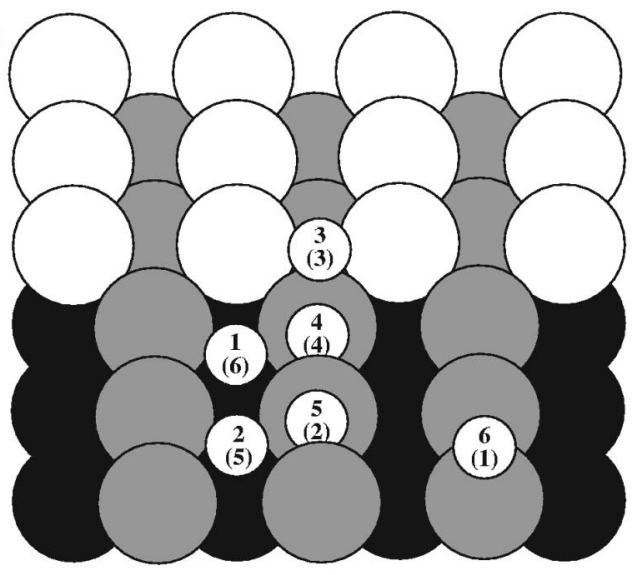

(b)

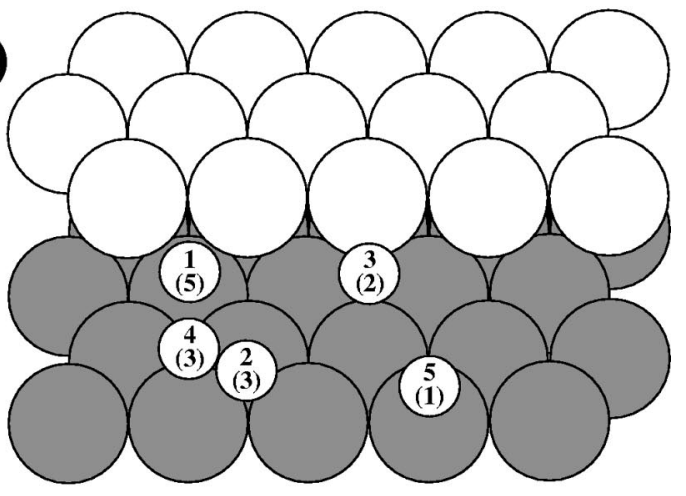

(d)

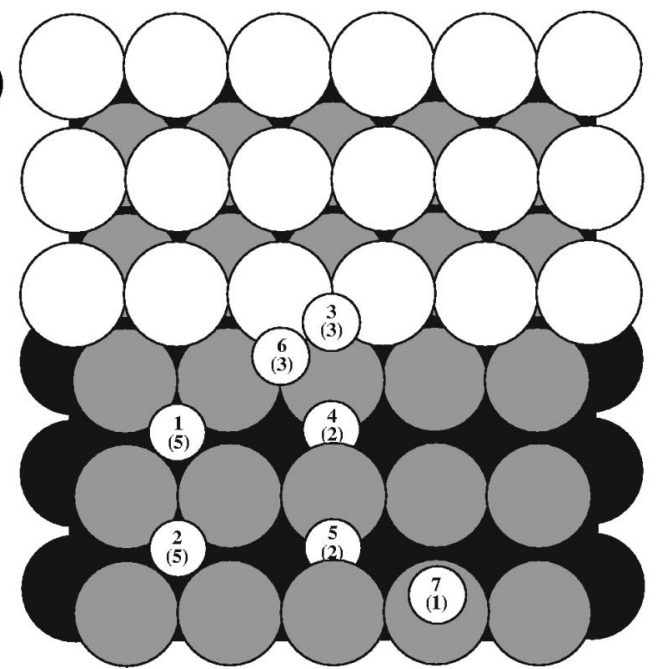

FIG. 1. Possible adsorption sites of $\mathrm{S}$ on (a) $\mathrm{Pd}(211)$, (b) $\mathrm{Pd}(331)$, (c) $\mathrm{Pd}(320)$, and (d) $\mathrm{Pd}(551)$. The first labels indicate the adsorption energy in a descending order (see also Table IV below) and the label in parentheses gives the coordination of the $\mathrm{S}$ atom at the particular site.

vicinals $\operatorname{Pd}(320)$ and $\operatorname{Pd}(551)$, there are five undercoordinated sites, three on the terrace and two underneath. These form the first five "layers." For comparison with the entries in Table II, we obtained the relaxation between two topmost layers $\Delta d_{1,2}=+0.3 \%$ and $-9.2 \%$ for $\operatorname{Pd}(111)$ and $\operatorname{Pd}(110)$, respectively, whereas the corresponding relaxations obtained by Methfessel et al. ${ }^{34}$ are $-0.1 \%$ and $-5.3 \%$.

To evaluate the effect of the thickness of the slab on the relaxation, we performed calculations for several layer thicknesses in each case and we found the calculations to be con-

TABLE II. The calculated percentage changes in interlayer relaxations for clean surfaces. The subscripts indicate the layers, 1 being the topmost one, etc. Changes are along the surface normal.

\begin{tabular}{lrrrr}
\hline \hline$\Delta d_{i, i+1}$ & $\operatorname{Pd}(211)$ & $\operatorname{Pd}(331)$ & $\operatorname{Pd}(320)$ & $\operatorname{Pd}(551)$ \\
\hline$\Delta d_{1,2}$ & $-12.3 \%$ & $-12.0 \%$ & $-15.5 \%$ & $-8.8 \%$ \\
$\Delta d_{2,3}$ & $-13.0 \%$ & $-7.7 \%$ & $-18.0 \%$ & $-15.7 \%$ \\
$\Delta d_{3,4}$ & $+17.4 \%$ & $+10.9 \%$ & $+1.8 \%$ & $-3.4 \%$ \\
$\Delta d_{4,5}$ & $-6.1 \%$ & $-4.8 \%$ & $-11.6 \%$ & $-6.5 \%$ \\
$\Delta d_{5,6}$ & $-2.0 \%$ & $+0.2 \%$ & $+22.8 \%$ & $+18.5 \%$ \\
$\Delta d_{6,7}$ & $+3.6 \%$ & & $-8.2 \%$ & $-6.9 \%$ \\
$\Delta d_{7,8}$ & $-2.7 \%$ & & $-3.1 \%$ & $-1.7 \%$ \\
$\Delta d_{8,9}$ & $+0.0 \%$ & & $+6.3 \%$ & $+2.8 \%$ \\
\hline \hline
\end{tabular}

verged with the thicknesses mentioned in Sec. II. For the two vicinals of $\operatorname{Pd}(110)$ the relaxations continue to be strong even beyond the ninth layer, while for $\operatorname{Pd}(211)$ and $\operatorname{Pd}(331)$, the relaxations die out after the seventh and the fourth layer, respectively. As a matter of fact, for $\operatorname{Pd}(320)$ the relaxations for $\Delta d_{9,10}$ and $\Delta d_{10,11}$ were as large as $-6.7 \%$ and $+4.6 \%$, respectively. It is thus important that relaxations of the inner layers of vicinals with different geometries be examined individually, with the rule of thumb that the more open the

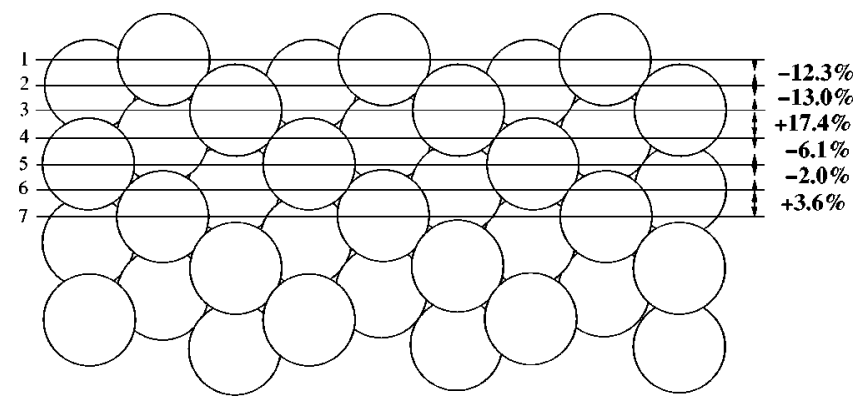

FIG. 2. Labeling of the layers and magnitudes of the interlayer relaxations for $\mathrm{Pd}(211)$. 
surface the larger the extent to which the inner layers are perturbed from their bulk terminated positions.

The relaxation patterns of the vicinals of $\operatorname{Pd}(111)$ and $\operatorname{Pd}(110)$ are different in one important way. While the first two interlayer spacings are largely contracted on all surfaces, the first expanded spacing is that between the third and the fourth layers, for the vicinals of $\mathrm{Pd}(111)$, and the fifth and the sixth, for those of $\operatorname{Pd}(110)$. Even though in our calculations, we found a small outward relaxation for the third/fourth layer of $\mathrm{Pd}(320)$, it is really the fifth/sixth layer spacing that has an impressive expansion. A look at the geometry of these surfaces shows that this difference in the results for vicinals of $\operatorname{Pd}(111)$ and $\operatorname{Pd}(110)$ is not surprising. Even though the terraces of the four vicinals considered here have three atoms each, the local coordination and geometry are different. As mentioned earlier, for $\operatorname{Pd}(211)$ and $\operatorname{Pd}(331)$, layers deeper than the third are not exposed to the vacuum and have coordination of 12 , while for $\operatorname{Pd}(320)$ and $\operatorname{Pd}(551)$, this situation arises after the fifth layer because of the more open geometry of the surface. Following Smoluchowski's ideas of charge smoothing, ${ }^{12}$ it is only natural to see that $\Delta d_{3,4}$ is positive for $\operatorname{Pd}(211)$ and $\operatorname{Pd}(331)$ and $\Delta d_{5,6}$ for $\operatorname{Pd}(320)$ and $\operatorname{Pd}(551)$. These trends in relaxation patterns are also in qualitative agreement with conclusions reached in a recent $a b$ initio study of vicinals of $\mathrm{Cu}(100)$ and $\mathrm{Cu}(111) .{ }^{13}$ The fact that the magnitude of the relaxations of the deeper layers is larger for the vicinals of $\operatorname{Pd}(110)$ is also not surprising because of the inherent openness of this structure and is reminiscent of the relatively larger relaxation of $\mathrm{Pd}(110)$ itself as compared to $\operatorname{Pd}(111)$.

A few earlier $a b$ initio studies of vicinal Pd surfaces exist, taking the atomic relaxations fully into account, see, e.g., Ref. 7. Relaxations have been considered for the steps on the $\mathrm{Pt}(111)$ surface $^{35,36}$ and on different $\mathrm{Cu}$ surfaces. ${ }^{13,37,38}$ For $\mathrm{Cu}(211)$ and $\mathrm{Cu}(331)$, Heid et al. ${ }^{13}$ obtained qualitatively similar relaxation patterns as we do for $\operatorname{Pd}(211)$ and $\operatorname{Pd}(331)$. Interestingly, for $\operatorname{Pd}(331)$, we obtain a similar $(--+)$ pattern as found in the embedded atom method (EAM) calculations. ${ }^{39}$ Also, for $\mathrm{Pd}(211)$, our relaxation pattern agrees with the EAM one. For $\mathrm{Cu}(331)$, the EAM (Refs. 39,40 ) has been shown to disagree both with pseudopotential $^{13}$ and full-potential linearized augmented plane-wave ${ }^{37}$ calculations. As noted in earlier comparisons for $\mathrm{Cu},{ }^{13,37}$ however, the absolute changes in the interlayer spacings vary between different computational approximations by more than a factor of 2 . This is the case even when comparing the different first-principles results with each other. It is, therefore, not meaningful to compare these numbers one by one with the EAM results, yet the trends seem to agree well. It should also be mentioned that for stepped surfaces, interlayer spacings are much smaller than those for their flat surface counterparts. The calculated changes in these spacings are thus even smaller and the margin of error large in measurements and in theoretical calculations.

On the surfaces considered here, experimental values of multilayer relaxations are available only for $\operatorname{Pd}(320)$ in the work of Hirsimäki et al. ${ }^{16}$ who have used low-energy electron-diffraction technique to obtain $-15 \%,-9 \%$, $-6 \%,+15 \%$, and $+6 \%$ for the relaxations of the successive layers. The major disagreement here is with the relax- ation of $\Delta d_{3,4}, \Delta d_{4,5}$, and $\Delta d_{5,6}$, for which we find $+1.8 \%$, $-11.6 \%$, and $+22.8 \%$, respectively. In an independent similar computational work, ${ }^{41}$ the following trend in the successive relaxations of $\operatorname{Pd}(320)$ is found: $-11.9 \%$, $-19.2 \%, \quad-0.6 \%,-5.9 \%, \quad+16.8 \%,-4.5 \%$, and $-3.2 \%$. These results suggest that in the analysis of the experimental data, a larger number of layers (they have allowed only six layers) needs to be allowed to relax before we can make a definite comparison.

The surface energies for the different low- and high-index surfaces have been calculated using

$$
E_{s}=\frac{1}{A}\left(E_{\text {slab }}^{r e l}-\frac{1}{2} E_{\text {slab }}^{\text {unrel }}-\frac{1}{2} N E_{0}\right)
$$

where $A$ is the area of the supercell, $E_{\text {slab }}^{r e l}$ and $E_{\text {slab }}^{\text {unrel }}$ is the energy of the relaxed and unrelaxed slab, respectively, $N$ is the number of atoms in the supercell, $E_{0}$ the energy per atom in the bulk, and the factor $1 / 2$ accounts for two surfaces in the slab. In the cases of the low-index surfaces, we find our values of $0.55 \mathrm{meV} / \mathrm{atom}\left(81.1 \mathrm{meV} / \AA^{2}\right)$ and $1.07 \mathrm{meV} /$ atom (96.1 meV/ $\left.\AA^{2}\right)$ for $\operatorname{Pd}(111)$ and $\operatorname{Pd}(110)$, respectively, showing a general trend that a close-packed surface has a smaller surface energy. These values are also in good agreement with the linear-muffin-tin-orbital (LMTO) work of Methfessel et al. ${ }^{34}$ whose corresponding values are 0.68 $\mathrm{meV} / \mathrm{atom}$ and $1.33 \mathrm{meV} / \mathrm{atom}$. Both Weinert et al. ${ }^{42}$ and Vitos et al. ${ }^{43}$ obtained somewhat larger surface energies. Quite obviously, atomic relaxations tend to lower the surface energies; in the above-mentioned references, only Methfessel et al. ${ }^{34}$ have taken them into account. Comparing the surface energies expressed in $\mathrm{meV} / \AA^{2}$, we obtain the ratio between the surface energies of $\operatorname{Pd}(110)$ and $\operatorname{Pd}(111)$ to be 1.2 , in good agreement with the prediction of the "liquid drop model" of Perdew et al. ${ }^{44}$ The factors that can cause differences in surface energies include the structure of the terrace face (111 vs 110), step to step distance, and the structure of the steps. For $\operatorname{Pd}(211), \operatorname{Pd}(331), \operatorname{Pd}(320)$, and $\operatorname{Pd}(551)$, our calculated surface energies are $92.1 \mathrm{meV} / \AA^{2}, 94.3 \mathrm{meV} / \AA^{2}$, $98.1 \mathrm{meV} / \AA^{2}$, and $94.3 \mathrm{meV} / \AA^{2}$, respectively. The systematic difference in surface energies between vicinals of $\operatorname{Pd}(111)$ and $\operatorname{Pd}(110)$ is most likely caused by the different structure of the terrace face as $\operatorname{Pd}(111)$ is energetically more favorable than $\operatorname{Pd}(110)$.

We have also calculated the step-formation energies using the formula ${ }^{45}$

$$
E_{\text {step }}=E_{\text {surf }}(\theta)-(n-1) E_{\text {surf }}(0)-f_{\text {step }} E_{\text {surf }}(0),
$$

where $E_{\text {surf }}(\theta)$ and $E_{\text {surf }}(0)$ are the surface energies per atom for the vicinal and flat surfaces, respectively. The index $n$ is the number of atoms in a terrace perpendicular to step edges and the values for the factors $f_{\text {step }}$ are tabulated in Ref. 45 . The step-formation energies are shown in Table III. They have been compared with the results of other calculations obtained using the tight-binding scheme ${ }^{46}$ and effective pair potentials introduced by Vitos et $a l^{45}$ and applied to their own LMTO database. ${ }^{43}$ There are several factors that might explain the differences between the step-formation energies, obtained by different groups in Table III, namely, accuracy of 
TABLE III. Step-formation energies in eV/atom. The results of Raouafi et al. were obtained using tight-binding calculations, ${ }^{46}$ and Vitos et al. used an effective pair-potential fit ${ }^{45}$ to LMTO data. ${ }^{43}$

\begin{tabular}{lccc}
\hline \hline & Present & Raouafi & Vitos \\
\hline$(211)$ & 0.30 & 0.425 & \\
$(331)$ & 0.33 & 0.432 & 0.460 \\
$(320)$ & 0.11 & & 0.230 \\
$(551)$ & -0.03 & 0.003 & 0.045 \\
\hline \hline
\end{tabular}

the method used, whether surface relaxations are taken into account, choice of values for $n$, etc. For instance, the usage of a pair-potential expansion for extracting the energy of vicinal surfaces, as opposed to employing calculated $a b$ initio values for the surface energies of low-index surfaces, may explain the difference between the results of Vitos et al. and ours. Incidently, using the value $E_{\text {coh }}=3.94 \mathrm{eV} /$ atom, ${ }^{47}$ we find the ratio between the formation energy of the (111) vicinals and the cohesive energy to be about $8 \%$ that is reasonably close to the "universal" value $7 \%$, calculated by Feibelman. $^{48}$ The step-formation energies for vicinals of $\mathrm{Pd}(110)$ in Table III is found to be remarkably small, particularly for $\operatorname{Pd}(551)$ for which it is zero (within the limits of errors in the calculations). The implications of this on the temperature-dependent behavior of these surfaces should be interesting, but to fully understand that vibrational entropy contributions need to also be taken into consideration which is beyond the scope of this work.

The ratios of the step-formation energies can also be used for predicting the shapes of islands on flat terraces. ${ }^{36}$ If the formation energies for $A$ - and $B$ - type steps on an fcc(111) surface are equal, the equilibrium island shape should be hexagonal for geometric reasons. On the other hand, it has been shown experimentally ${ }^{49}$ and theoretically ${ }^{36}$ for $\mathrm{Pt}$ and also predicted for Al (Ref. 50) that the (111)-faceted steps are preferred, thus leading to the formation of nonhexagonal islands. For the steps on $\operatorname{Pd}(111)$, we find the ratio of the step-formation energies to be 1.10, suggesting the possibility of nonhexagonal island formation. Interestingly, in this case, the (100)-faceted step seems to be favored, in contrast to what has been observed for Pt. These considerations too do not take into account the effect of vibrational entropy that is also expected to play a role at finite temperatures. ${ }^{40}$

\section{S ADSORPTION ON STEPPED Pd SURFACES}

The sites where the $\mathrm{S}$ atom was found to adsorb are depicted in Fig. 1. The corresponding adsorption energies are shown in Table IV. Because of the narrow supercell, there is actually always a chain of atoms on the surface. The distance between adjacent $\mathrm{S}$ atoms in the chain is $3.96 \AA$ on $\operatorname{Pd}(320)$ and $2.80 \AA$ on the other surfaces. $\mathrm{S}$ was found to adsorb in every hollow site on the terrace face of $\operatorname{Pd}(211)$ and $\operatorname{Pd}(331)$, the vicinals of $\mathrm{Pd}(111)$. The fcc sites are more stable than the hcp sites, and sites below the step edge are more favorable than similar sites located higher on the terrace face. There were two top sites found on $\operatorname{Pd}(211)$ and one on $\operatorname{Pd}(331)$, and one bridge site on both surfaces. As expected, hollow
TABLE IV. S adsorption energies in $\mathrm{eV}$ at different sites on vicinal Pd surfaces (see also Fig. 1). The coordination numbers at the different sites are given in parentheses.

\begin{tabular}{lcccc}
\hline \hline Site & $\operatorname{Pd}(211)$ & $\operatorname{Pd}(331)$ & $\operatorname{Pd}(320)$ & $\operatorname{Pd}(551)$ \\
\hline 1 & $-4.44(4)$ & $-4.60(5)$ & $-5.16(6)$ & $-4.67(5)$ \\
2 & $-4.36(3)$ & $-4.48(3)$ & $-4.99(5)$ & $-4.63(5)$ \\
3 & $-4.31(4)$ & $-4.34(2)$ & $-4.96(3)$ & $-4.45(3)$ \\
4 & $-4.30(3)$ & $-4.24(3)$ & $-4.58(4)$ & $-4.33(2)$ \\
5 & $-3.73(2)$ & $-3.40(1)$ & $-4.17(2)$ & $-4.33(2)$ \\
6 & $-3.33(1)$ & & $-2.89(1)$ & $-4.21(3)$ \\
7 & $-3.27(1)$ & & & $-3.34(1)$ \\
\hline \hline
\end{tabular}

sites are the most stable sites before bridge and top sites, respectively.

On the vicinals of $\operatorname{Pd}(110), \operatorname{Pd}(320)$, and $\operatorname{Pd}(551), S$ was found to adsorb in two hollow and two bridge sites on the terrace face. In addition, there was one hollow site on the step face of $\operatorname{Pd}(320)$, two hollow sites on the step face of $\operatorname{Pd}(551)$, and one top site on the step chain on both surfaces. Again, the hollow sites are the most and top sites the least stable sites. The sites below the step edge are also more favorable than the similar sites higher on the terrace face. The fcc site on the step face of $\operatorname{Pd}(551)$ is more stable than the hcp site. The adsorption energy of $-5.16 \mathrm{eV}$ for $\operatorname{Pd}(320)$ makes it the most strongly receptive to $\mathrm{S}$ adsorption. The binding energies for $S$ on the other three vicinal surfaces have very similar range, between $-4.6 \mathrm{eV}$ and $-3.3 \mathrm{eV}$. For comparison, the adsorption energies of $\mathrm{S}$ in hollow sites are found to be $-3.25 \mathrm{eV}$ for $\mathrm{Pd}(111)$ and $-4.43 \mathrm{eV}$ for $\operatorname{Pd}(110)$ for full coverage.

The adsorbed chain of $\mathrm{S}$ atoms affects the relaxation of the surfaces. The changes in coordinates between a clean relaxed surface and a surface with a chain of $\mathrm{S}$ atoms are larger than the displacements of atoms between unrelaxed and relaxed clean surfaces. The symmetry of the supercells prevents relaxations in $x$ direction (along the step chains). The larger coverage of $\mathrm{S}$ on $\mathrm{Pd}(211)$ causes restrictions in the relaxation. There is $\mathrm{S}$ on every step, which means that the relaxation of the steps is identical. On all the other surfaces, there is $\mathrm{S}$ on every second step and the surfaces can relax more freely. When the $\mathrm{S}$ atom chain is placed on the surface, the nearest $\mathrm{Pd}$ atoms generally move away from the $\mathrm{S}$ chain in the $y$ direction (the direction perpendicular to the step chains in the surface plane). In general, the adsorption site of the $\mathrm{S}$ atoms does not have much effect on how the layers of the surfaces relax in the $z$ direction. The most pronounced relaxation is on $\operatorname{Pd}(320)$ in which, for adsorption site 4 [see Fig. 1(c)], the closest step atoms relax by about $0.71 \AA$ and $0.35 \AA$ along the $y$ and $z$ directions, respectively. This is interesting because the coordination of the step atoms on $\operatorname{Pd}(320)$ is 6 while on the other three surfaces it is 7 . The displacements of the nearby $\mathrm{Pd}$ atoms induced by $\mathrm{S}$ adsorption on $\mathrm{Pd}(551)$ are shown in Table $\mathrm{V}$. The $\mathrm{S}$ induced relaxations on the other three surfaces are similar and we have not included them for brevity.

The effect of the coordination on the preferred adsorption sites is also reflected in the electronic structure. In Fig. 3, we 
TABLE V. Relaxations (in $\AA$ ) induced on Pd atoms by S on the Pd(551) surface at different sites for S [see Fig. 1(d)]. Subscript denotes the layer index, $y$ direction is perpendicular to the step edge and $z$ direction is the normal to the surface.

\begin{tabular}{lccccccc}
\hline \hline Site: & 1 & 2 & 3 & 4 & 5 & 6 & 7 \\
\hline$\Delta y_{1}$ & -0.08 & -0.13 & -0.21 & -0.05 & -0.10 & -0.23 & -0.02 \\
$\Delta z_{1}$ & +0.06 & +0.18 & +0.23 & +0.01 & +0.13 & +0.16 & +0.10 \\
$\Delta y_{2}$ & -0.06 & -0.01 & +0.07 & -0.07 & +0.07 & +0.03 & $\sim 0$ \\
$\Delta z_{2}$ & +0.19 & -0.01 & $\sim 0$ & +0.14 & +0.15 & -0.01 & +0.01 \\
$\Delta y_{3}$ & +0.03 & +0.04 & +0.04 & +0.06 & -0.07 & +0.07 & $\sim 0$ \\
$\Delta z_{3}$ & +0.18 & $\sim 0$ & -0.05 & +0.09 & +0.05 & +0.09 & +0.01 \\
$\Delta y_{4}$ & -0.08 & +0.01 & +0.01 & -0.06 & +0.01 & -0.02 & $\sim 0$ \\
$\Delta z_{4}$ & +0.06 & $\sim 0$ & $\sim 0$ & +0.04 & -0.08 & -0.02 & +0.02 \\
$\Delta y_{5}$ & +0.07 & +0.09 & +0.09 & +0.05 & +0.07 & +0.05 & -0.01 \\
$\Delta z_{5}$ & -0.14 & +0.10 & -0.02 & -0.08 & +0.06 & -0.03 & -0.02 \\
\hline \hline
\end{tabular}

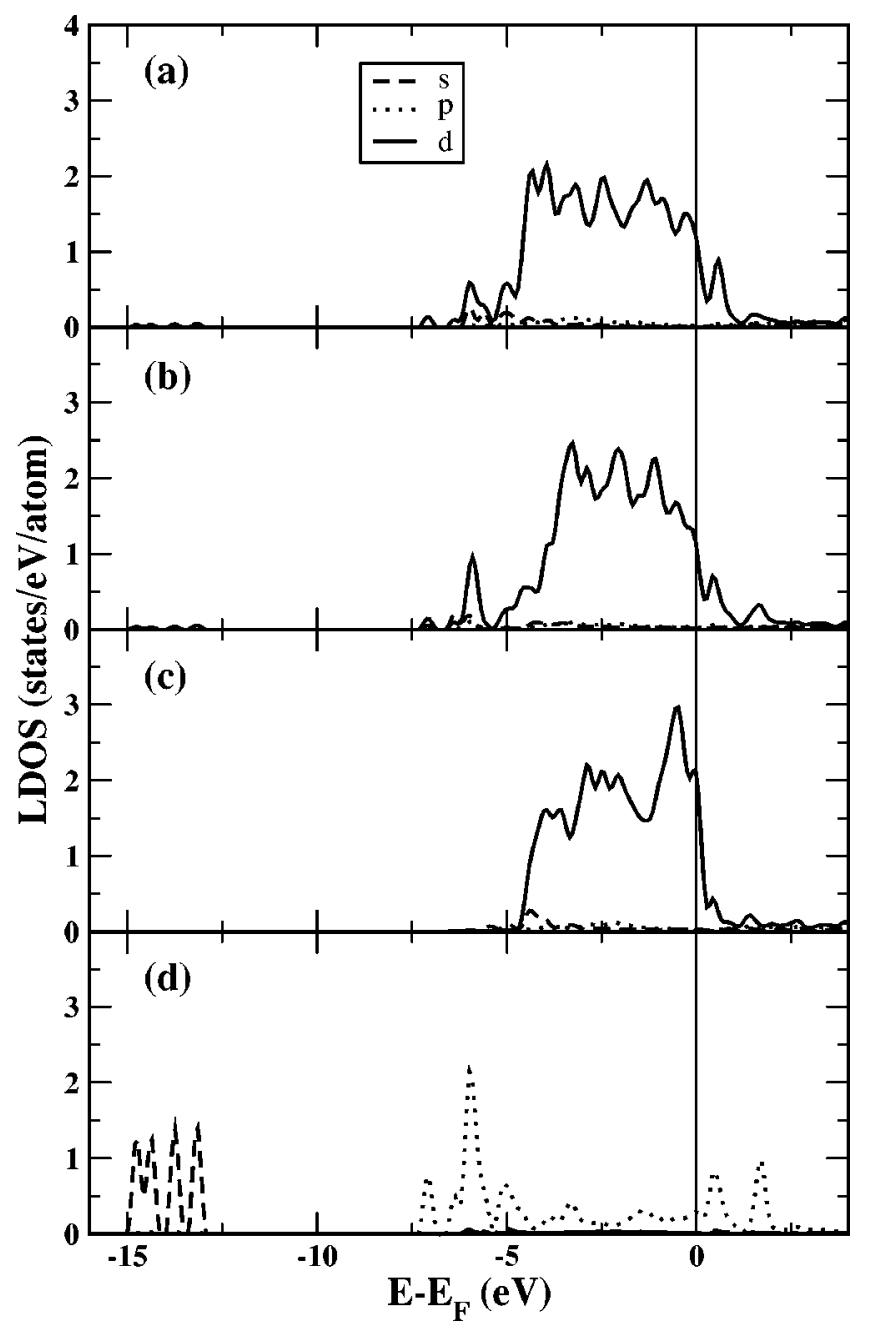

FIG. 3. For the $\operatorname{Pd}(551)$ surface, $\mathrm{S}$ at the preferred adsorption site [denoted 1 in Fig. 1(d)]: Angular momentum decomposition of LDOS for (a) a Pd atom, just below the $\mathrm{S}$ atom, (b) the next-nearest $\mathrm{Pd}$ atom that is the one below the step edge, (c) a Pd atom, further from the $S$ atom, and (d) the $S$ atom at site 1 . The Fermi level is at zero energy.

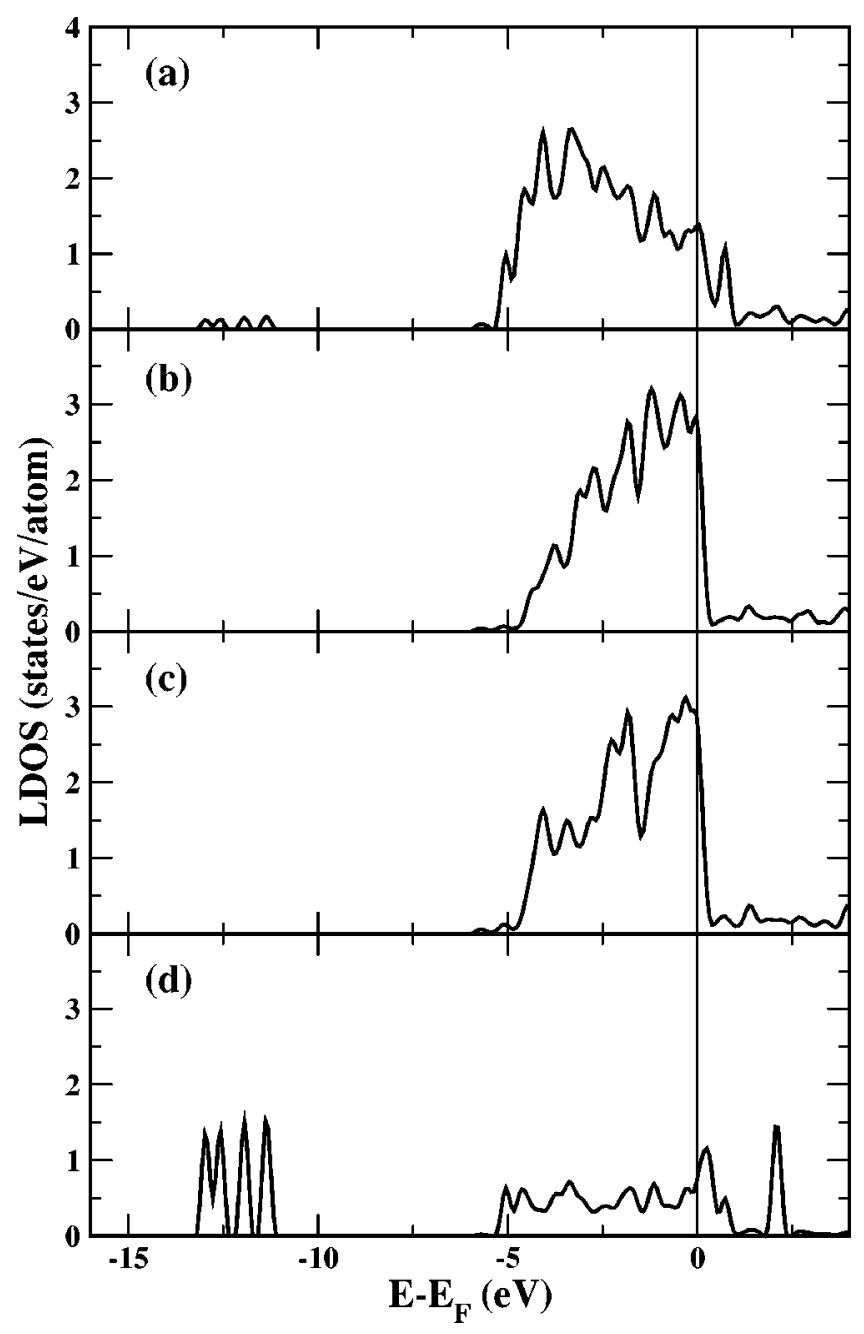

FIG. 4. For the $\mathrm{Pd}(551)$ surface, $\mathrm{S}$ at the least favored adsorption site [denoted 7 in Fig. 1(d)]: (a) LDOS at the site of a Pd atom, just below the $\mathrm{S}$ atom, (b) same for a $\mathrm{Pd}$ atom on the terrace face next to S, (c) LDOS for a Pd atom below the step edge, roughly at the same distance as the one in (b), and (d) LDOS for the S atom at site 7 . The Fermi level is at zero energy. 


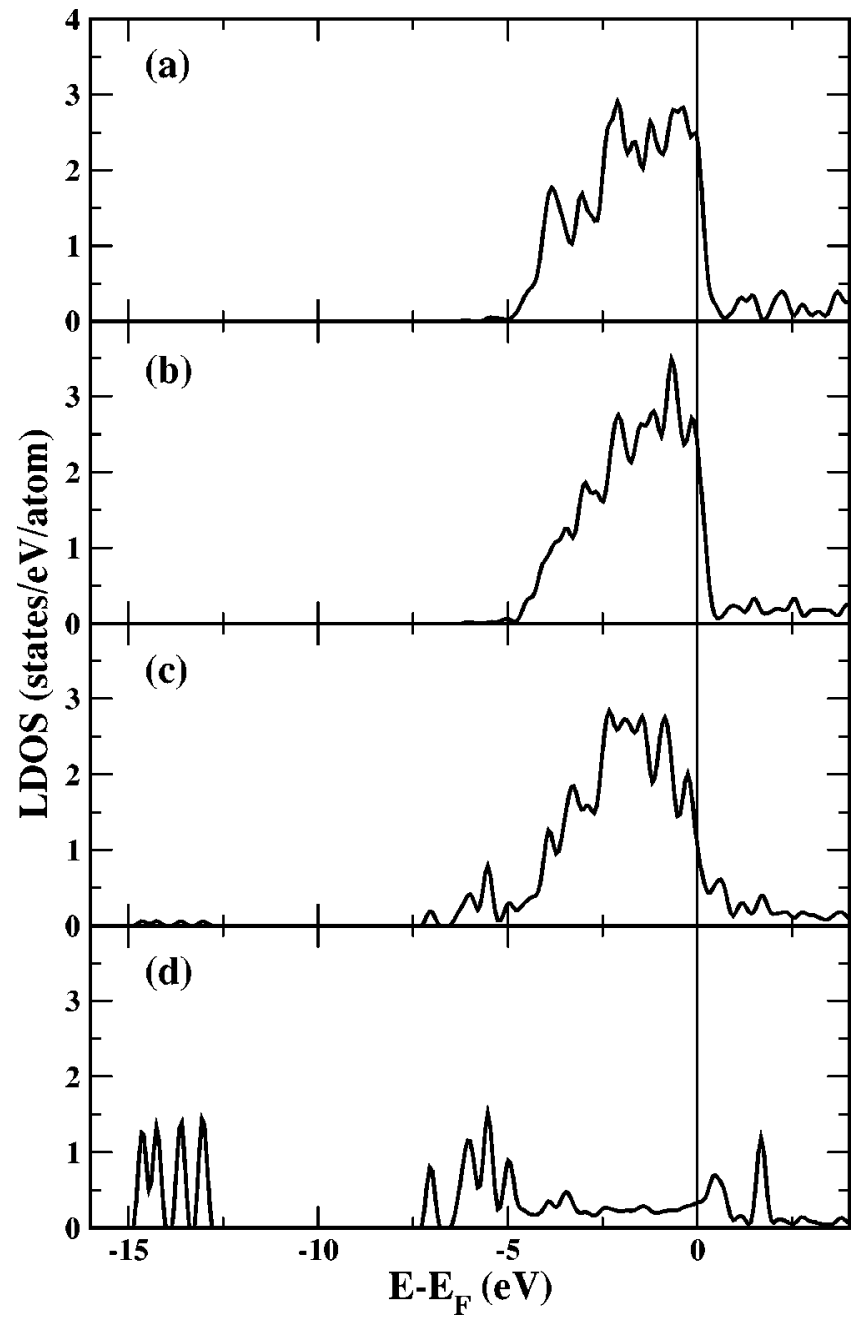

FIG. 5. LDOS for (a) an atom in the surface layer of the clean $\operatorname{Pd}(111)$, (b) atom at the step edge on the clean $\operatorname{Pd}(211)$, (c) the same step-edge $\mathrm{Pd}$ atom in the presence of a $\mathrm{S}$ atom in position 1 [defined in Fig. 1(a)] next to it, and (d) the $S$ atom in this position. The Fermi level is at zero energy.

represent the local densities of states (LDOS) for different atoms in the $\mathrm{Pd}(551)$ surface system. One can see that when the $\mathrm{S}$ atom on $\operatorname{Pd}(551)$ is located near the step edge, bond formation (seen as a similar character of LDOS below $-5 \mathrm{eV}$ ) occurs between $\mathrm{S}$ and its nearest neighbor atoms, one of which is at the distance $2.30 \AA$ from the S [LDOS in Fig. 3(a)] and other two at the distance $2.45 \AA$ [LDOS in Fig. 3(b) ]. Only further away the bond formation has almost completely vanished, see Fig. 3(c). On the other hand, when the $\mathrm{S}$ is at the top site as is the case in Fig. 4, there is only one nearest-neighbor atom with which the bond is formed. This situation is not energetically favorable and, therefore, the absolute value of the adsorption energy at this site is clearly smaller than that at the position close to the step edge.

The electronic structures can be further analyzed by looking at the state projected LDOS's, i.e., the angular momentum decomposition in Fig. 3 for $\mathrm{Pd}(551)$. It can be seen that the lowest lying states for $\mathrm{S}$ are, as expected, $s$-like and are some $12-15 \mathrm{eV}$ below the Fermi level. Some couplings between these states and the $\mathrm{Pd} d$-states can be seen at the

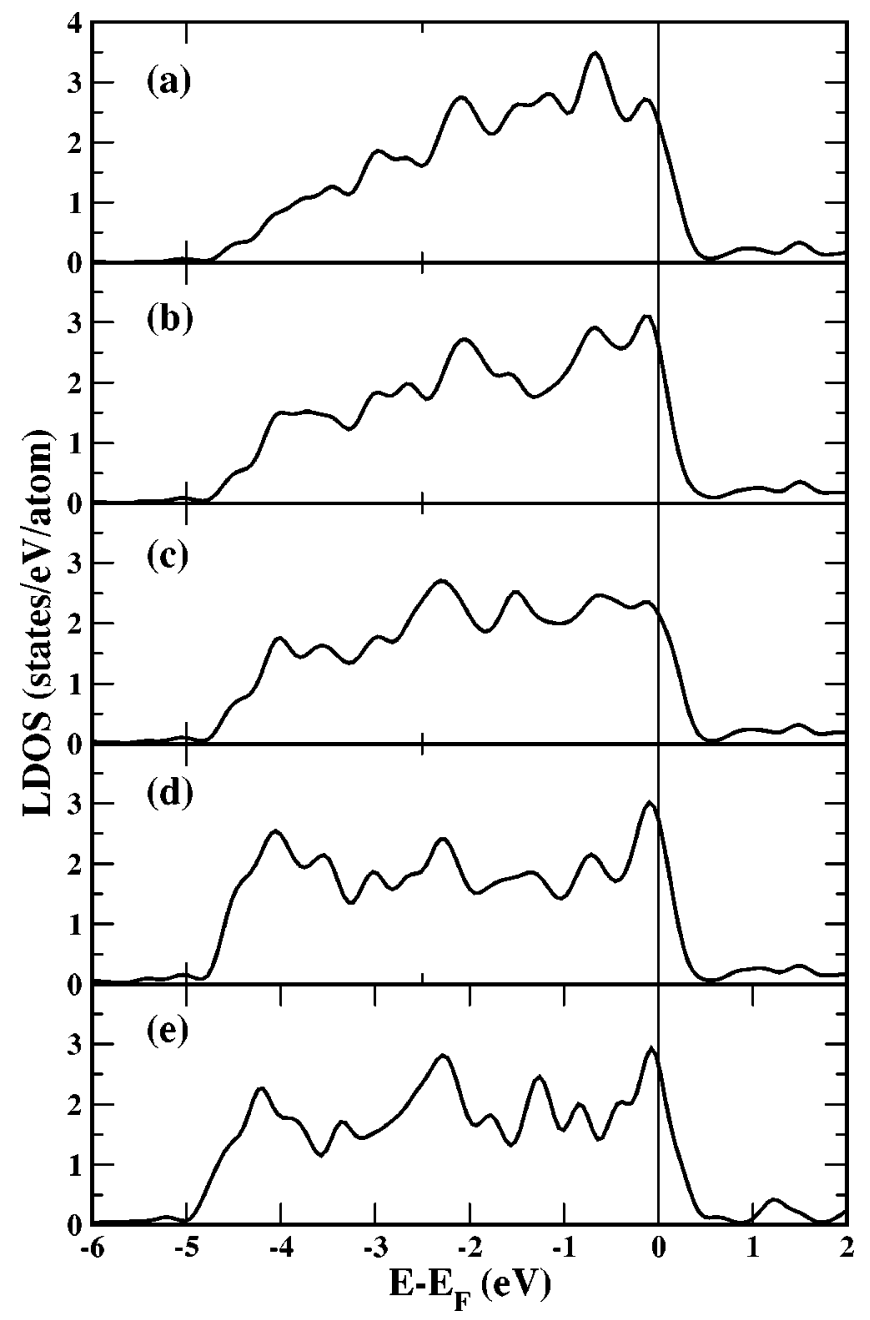

FIG. 6. LDOS for the atoms in different layers on the clean $\operatorname{Pd}(211)$ surface, from the first (a) to the fourth layer (d), and in the bulk (e). The Fermi level is at zero energy.

LDOS for the $\mathrm{Pd}$ atoms close to the $\mathrm{S}$ atom. The $\mathrm{S} p$-states that have bonding peaks at and below $5 \mathrm{eV}$ below the Fermi level are clearly coupled to the closest Pd atoms, whereas further away from the $\mathrm{S}$ atom this coupling has completely died out. Moreover, when $\mathrm{S}$ is at the top site, there is no clear bonding peak below the Fermi level in the LDOS at the $\mathrm{S}$ site [see Fig. 4(d)], whereas the antibonding states above the Fermi level are even more pronounced than the ones in Fig. 3(d) for $\mathrm{S}$ near the step edge.

On the Pd(111) surface, at low coverages, $\mathrm{S}$ is known to form ordered structures. ${ }^{51,52}$ The search of such structures would require addressing much lower coverages than in the present work, which using the method at hand would need prohibitively large supercells. Nevertheless, the fact that the $\mathrm{S}$ atoms prefer positions close to the steps suggests the possible formation of $\mathrm{S}$ stripes along the step edges.

The electronic structures of the stepped surfaces, with and without $\mathrm{S}$, can be analyzed by comparing the LDOS in the different cases. For the clean surfaces, an interesting question is that of the possible formation of one-dimensional (1D) surface states that have been seen in the experiments for stepped $\mathrm{Cu}$ surfaces. ${ }^{53-55}$ While it is difficult to draw definite 
conclusions about the 1D nature of such states based on the present calculations, one can see a clear difference between the LDOS for a step-edge atom on the $\operatorname{Pd}(211)$ surface and an atom in the surface layer of the smooth $\operatorname{Pd}(111)$ surface. These are depicted in Fig. 5. In the LDOS of Pd(211), there is a strong peak about $0.7 \mathrm{eV}$ below the Fermi level, which does not exist in the case of the (111) surface. That this is indeed a surface state or a surface resonance, can be seen in Fig. 6, where the LDOS for $\mathrm{Pd}(211)$ is plotted layer by layer. The state decays when going deeper in the slab and finally, as expected, the LDOS ends up resembling that in the bulk. Also shown in Fig. 5 is the effect of S. The LDOS of the Pd atom at the step edge site is clearly reduced at the Fermi level which is an indication of reduction of the catalytic activity. Moreover, the center of the $d$-band is shifted down in energy which also indicates reduced reactivity. ${ }^{56}$ The amplitude of the above-mentioned state diminishes considerably in the presence of S. Again, the bond between the $\mathrm{S}$ atom and the surface Pd can be seen as peaks about $5 \mathrm{eV}$ below the Fermi level in both the $\mathrm{S}$ and Pd LDOS.

\section{CONCLUSIONS}

In conclusion, we have performed a first-principles study for the structure and relaxations of clean and $\mathrm{S}$ covered vici- nal Pd surfaces. The trends in the relaxation patterns of the clean Pd vicinals considered here are in accordance with the rationale presented in earlier works on other stepped surfaces. On comparing our results on $\mathrm{Pd}(320)$ with recent experimental data, we find disagreements that may be related to the number of layers allowed to relax in the analysis of the experimental data. Adsorbed $\mathrm{S}$ was shown to affect the relaxations considerably. The presence of $\mathrm{S}$ also causes remarkable changes in the electronic structure, which is seen as modifications of the LDOS and reduction of it at the Fermi level. This provides further evidence to the fact that the poisoning effect is due to the changes in the electronic structure, induced by $\mathrm{S}$ adsorption.

\section{ACKNOWLEDGMENTS}

We acknowledge the generous computer resources of CSC-Scientific Computing Ltd., Espoo, Finland. This work has been supported in part by the Academy of Finland through its Center of Excellence program and by the U.S. National Science Foundation under Grant No. CHE-0205064. T.S.R. thanks colleagues at the Laboratory of Physics, Helsinki University of Technology, for their warm hospitality during her stay there.
${ }^{1}$ T. Zambelli, J. Wintterlin, J. Trost, and G. Ertl, Science 273, 1688 (1996).

${ }^{2}$ B. Hammer, Phys. Rev. Lett. 83, 3681 (1999).

${ }^{3}$ G.A. Somorjai, Introduction to Surface Chemistry and Catalysis (Wiley, New York, 1994).

${ }^{4}$ P. Gambardella, Z. Sljivancanin, B. Hammer, M. Blanc, K. Kuhnke, and K. Kern, Phys. Rev. Lett. 87, 056103 (2001).

${ }^{5}$ C.E. Tripa and J.T. Yates, Jr., J. Chem. Phys. 115, 8552 (2001).

${ }^{6}$ L. Savio, L. Vattuone, and M. Rocca, Phys. Rev. Lett. 87, 276101 (2001).

${ }^{7}$ M. Lischka and A. Groß, Phys. Rev. B 65, 075420 (2002).

${ }^{8}$ K. Irokawa, S. Ito, T. Kioka, and H. Miki, Surf. Sci. 433-435, 297 (1999).

${ }^{9}$ B. Hammer and J.K. Nørskov, Phys. Rev. Lett. 79, 4441 (1997).

${ }^{10}$ R.D. Ramsier, Q. Gao, H. Neergard Waltenburg, K.-W. Lee, O.W. Nooij, L. Lefferts, and J.T. Yates, Jr., Surf. Sci. 320, 209 (1994).

${ }^{11}$ S. Dahl, A. Logadottir, R.C. Egeberg, J.H. Larsen, I. Chorkendorff, E. Törnqvist, and J.K. Nørskov, Phys. Rev. Lett. 83, 1814 (1999).

${ }^{12}$ R. Smoluchowski, Phys. Rev. 60, 661 (1941).

${ }^{13}$ R. Heid, K.P. Bohnen, A. Kara, and T.S. Rahman, Phys. Rev. B 65, 115405 (2002).

${ }^{14}$ U. Muschiol, P.K. Schmidt, and K. Christmann, Surf. Sci. 395, 182 (1998).

${ }^{15}$ P.K. Schmidt, K. Christmann, G. Kresse, J. Hafner, M. Lischka, and A. Groß, Phys. Rev. Lett. 87, 096103 (2001).

${ }^{16}$ M. Hirsimäki, T. Pitkänen, M. Valden, M. Lindroos, and C.J. Barnes, Surf. Sci. 454-456, 6 (2000).

${ }^{17}$ Q. Gao, R.D. Ramsier, H. Neergaard Waltenburg, and J.T. Yates, J. Am. Chem. Soc. 116, 3901 (1994).
${ }^{18}$ M. Valden (private communication).

${ }^{19}$ F. Raouafi, C. Barreteau, D. Spanjaard, and M.C. Desjonquères, Phys. Rev. B 66, 045410 (2002).

${ }^{20}$ M. Rutkowski, D. Wetzig, and H. Zacharias, Phys. Rev. Lett. 87, 246101 (2001), and references therein.

${ }^{21}$ J.A. Rodriguez, J. Hrbek, M. Kuhn, S. Chaturvedi, and A. Maiti, J. Chem. Phys. 113, 11284 (2000).

${ }^{22}$ Z. Yang, R. Wu, and J.A. Rodriguez, Phys. Rev. B 65, 155409 (2002).

${ }^{23}$ P.J. Feibelman and D.R. Hamann, Phys. Rev. Lett. 52, 61 (1984).

${ }^{24}$ S. Wilke and M. Scheffler, Phys. Rev. Lett. 76, 3380 (1996); C.M. Wei, A. Groß, and M. Scheffler, Phys. Rev. B 57, 15572 (1998); A. Groß, and M. Scheffler, ibid. 61, 8425 (2000).

${ }^{25}$ X. Xiao, Y. Xie, C. Jakobsen, and Y.R. Shen, Phys. Rev. B 56, 12529 (1997); C.L. Fu and A.J. Freeman ibid. 40, 5359 (1989); E. Wimmer, C.L. Fu, and A.J. Freeman, Phys. Rev. Lett. 55, 2618 (1985).

${ }^{26}$ P. Hohenberg and W. Kohn, Phys. Rev. 136, B864 (1964); W. Kohn and L.J. Sham, ibid. 140, A1133 (1965).

${ }^{27}$ G. Kresse and J. Hafner, Phys. Rev. B 47, 558 (1993); G. Kresse and J. Furthmüller, Comput. Mater. Sci. 6, 15 (1996); G. Kresse and J. Furthmüller, Phys. Rev. B 54, 11169 (1996).

${ }^{28}$ D. Vanderbilt, Phys. Rev. B 41, 7892 (1990).

${ }^{29}$ G. Kresse and J. Hafner, J. Phys.: Condens. Matter 6, 8245 (1994).

${ }^{30}$ H.J. Monkhorst and J.D. Pack, Phys. Rev. B 13, 5188 (1976).

${ }^{31}$ M. Methfessel and A.T. Paxton, Phys. Rev. B 40, 3616 (1989).

${ }^{32}$ J.P. Perdew, in Electronic Structure of Solids '91, edited by P. Ziesche and H. Eschrig (Academic Verlag, Berlin, 1991).

${ }^{33}$ R. Lang, R.W. Joyner, and G.A. Somorjai, Surf. Sci. 30, 440 
(1972); for an introduction, see, G.A. Somorjai, Introduction to Surface Chemistry and Catalysis (Wiley, New York, 1994).

${ }^{34}$ M. Methfessel, D. Hennig, and M. Scheffler, Phys. Rev. B 46, 4816 (1992).

${ }^{35}$ P.J. Feibelman, Phys. Rev. B 52, 16845 (1995).

${ }^{36}$ G. Boisvert, L.J. Lewis, and M. Scheffler, Phys. Rev. B 57, 1881 (1998).

${ }^{37}$ W.T. Geng and A.J. Freeman, Phys. Rev. B 64, 115401 (2001).

${ }^{38}$ C.Y. Wei, S.P. Lewis, E.J. Mele, and A.M. Rappe, Phys. Rev. B 57, 10062 (1998).

${ }^{39}$ I.Yu. Sklyadneva, G.G. Rusina, and E.V. Chulkov, Surf. Sci. 416, 17 (1998).

${ }^{40}$ S. Durukanoglu, A. Kara, and T.S. Rahman, Phys. Rev. B 55, 13894 (1997).

${ }^{41}$ A. Kara (unpublished).

${ }^{42}$ M. Weinert, R.E. Watson, J.W. Davenport, and G.W. Fernando, Phys. Rev. B 39, 12585 (1989).

${ }^{43}$ L. Vitos, A.V. Ruban, H.L. Skriver, and J. Kollár, Surf. Sci. 411, 186 (1998).

${ }^{44}$ J.P. Perdew, Y. Wang, and E. Engel, Phys. Rev. Lett. 66, 508 (1991).

${ }^{45}$ L. Vitos, H.L. Skriver, and J. Kollár, Surf. Sci. 425, 212 (1999).

${ }^{46}$ F. Raouafi, C. Barreteau, M.C. Desjonquères, and D. Spanjaard,
Surf. Sci. 482-484, 1413 (2001).

${ }^{47}$ C. Kittel, Introduction to Solid State Physics (Wiley, New York, 1971).

${ }^{48}$ P.J. Feibelman, Phys. Rev. B 60, 11118 (1999).

${ }^{49}$ T. Michely and G. Comsa, Surf. Sci. 256, 217 (1991); T. Michely, M. Hohage, M. Bott, and G. Comsa, Phys. Rev. Lett. 70, 3943 (1993).

${ }^{50}$ R. Stumpf and M. Scheffler, Phys. Rev. B 53, 4958 (1996).

${ }^{51}$ V.R. Dhanak, A.G. Shard, B.C.C. Cowie, and A. Santoni, Surf. Sci. 410, 321 (1998).

${ }^{52}$ S. Speller, T. Rauch, J. Bömermann, P. Bormann, and W. Heiland, Surf. Sci. 441, 107 (1999).

${ }^{53}$ O. Sánchez, J.M. García, P. Segovia, J. Alvarez, A.L. Vázquez de Parga, J.E. Ortega, M. Prietsch, and R. Miranda, Phys. Rev. B 52, 7894 (1995).

${ }^{54}$ F. Baumberger, T. Greber, and J. Osterwalder, Phys. Rev. B 62, 15431 (2000).

${ }^{55}$ X.J. Shen, H. Kwak, D. Mocuta, A.M. Radojevic, S. Smadici, and R.M. Osgood, Jr., Phys. Rev. B 63, 165403 (2001).

${ }^{56}$ B. Hammer and J.K. Norskov, in Chemisorption and Reactivity on Supported Clusters and Thin Films, edited by R.M. Lambert and G. Pacchioni (Kluwer Academic, The Netherlands, 1997), pp. 285-351. 